\title{
Bioconvection of Nanofluid Past Stretching Sheet in a Porous Medium in Presence of Gyrotactic Microorganisms with Newtonian Heating
}

\author{
Rashid Pourrajab ${ }^{1}$ and Aminreza Noghrehabadi ${ }^{2}$ \\ ${ }^{1}$ Department of Mechanical Engineering, Shohadaye Hoveizeh University of Technology, Khuzestan, Iran \\ ${ }^{2}$ Department of Mechanical Engineering, Shahid Chamran University of Ahvaz, Ahvaz, Iran
}

\begin{abstract}
In this study, the effect of Newtonian heating on the boundary layer flow and heat transfer over a stretching surface in a porous medium in the presence of gyrotactic microorganisms and nanoparticle fractions are analysed. The governing equations are reduced to a system of couple non-linear ordinary differential equations, subjected to the Boussinesq approximation and asymmetric heat conditions. The reduced governing ordinary differential equations are then solved numerically. The solutions obtained are graphically represented. The effects of the controlling parameters on the flow, heat, nanoparticle concentration and the density of motile microorganisms have been examined. The results of the present study show the flow velocity, heat and mass transfer and motile microorganism characteristics on the stretching sheet are strongly influenced by the bioconvection parameters and Newtonian.
\end{abstract}

\section{Introduction}

The concept of bioconvection and nanofluid in biological porous media which is the emphasis of current work has many applications in medical engineering and biotechnological systems [1,2]. Nanofluid is considered as a fluid in which solid nanoparticles with the length scales of 1-100 nm are suspended in conventional heat transfer basic fluid. It has many practical applications in several engineering processes and medical sciences, such heat pipes [3], biofuel cells [4] and biomimetic microsystems [5]. Many researchers have focused on modeling the thermal conductivity and examined different viscosities of nanofluids over the past decade. The addition of nanoparticles to base fluids to improve their thermal conductivity and enhance the heat transfer performance was first demonstrated by Choi [6]. Several experimental and numerical analyses about nanofluid heat transfer performance can be found in Refs. [7-14].

Recently, the Buongiorno's model [15], who considered seven slip mechanisms which can produce a relative velocity between the nanoparticles and the base fluid, was further developed by Kuznetsov [16] to study the influence of using motile microorganisms for enhancing mass transport, inducing mixing and prevent nanoparticle agglomeration in nanofluids. Bioconvection in nanofluids occurs if the concentration of nanoparticles is little so that nanoparticles do not cause any significant increase in the viscosity of the base fluid. Nield and Kuznetsov [17, 18] used the mathematical nanofluid model proposed by Buongiorno to study the boundary layer flow of nanofluid in a porous medium. To the best of authors' knowledge, there is not any investigation to address the effect of the Newtonian heating boundary condition on the heat transfer characteristics of bioconvection of nanofluid flow over stretching sheet in a porous medium. The present study aims to examine the effect of the Newtonian heating boundary condition on the heat transfer characteristics of stretching sheet in the presence of nanoparticles and their dynamic effects.

\section{Governing equations}

Consider a two-dimensional incompressible and steady state viscous flow past a convectively heated vertical flat plate embedded in a porous medium filled with nanofluid containing gyrotactic microorganisms. It is assumed that there is no nanoparticle agglomeration, and the porous matrix does not absorb microorganisms and that the pore sizes are significantly larger than the microorganisms. Also, the presence of nanoparticles was assumed to have no effect on the direction in which microorganisms.

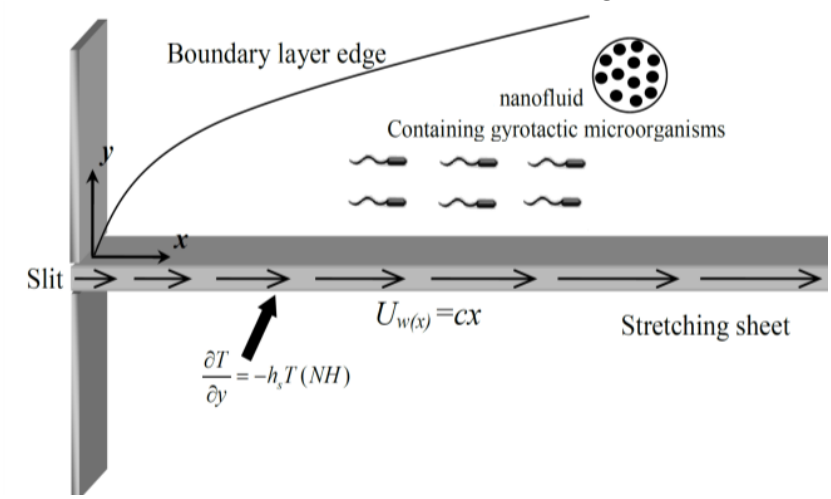

Figure 1. The physical sketch and coordinate system. 
The velocity of the surface is linear and is taken as $\mathrm{U}_{\mathrm{w}(\mathrm{x})}=\mathrm{c} . \mathrm{x}$ where $\mathrm{c}$ is a constant, and $\mathrm{x}$ is the coordinate component measured along the stretching surface. The physical model and coordinate system of this problem are shown in Figure 1.

We assume that the wall is subjected to a Newtonian heating boundary condition $(\mathrm{NH})$. Using scale analysis and applying the boundary layer approximations, the governing equations can be written as [19]:

$$
\begin{gathered}
\rho_{f}\left(u \frac{\partial u}{\partial x}+v \frac{\partial u}{\partial y}\right)=-\frac{\partial p}{\partial x}+\mu\left(\frac{\partial^{2} u}{\partial x^{2}}+\frac{\partial^{2} u}{\partial y^{2}}\right) \\
-\sigma B_{0}^{2} u-\frac{\mu u}{\kappa}+g\left[\begin{array}{l}
\left(1-C_{\infty}\right) \rho_{f \infty} \beta\left(T-T_{\infty}\right) \\
-\left(\rho_{p}-\rho_{f \infty}\right)\left(C-C_{\infty}\right) \\
-\left(\mathrm{n}-\mathrm{n}_{\infty}\right) \lambda\left(\rho_{m \infty}-\rho_{f}\right)
\end{array}\right] \\
u \frac{\partial T}{\partial x}+v \frac{\partial T}{\partial y}=\alpha\left(\frac{\partial^{2} T}{\partial y^{2}}\right)+\tau\left\{\begin{array}{l}
D_{B}\left(\frac{\partial C}{\partial y} \frac{\partial T}{\partial y}\right)+ \\
\frac{D_{T}}{T_{\infty}}\left[\left(\frac{\partial T}{\partial y}\right)^{2}\right]
\end{array}\right\}+ \\
\frac{\mu \alpha}{k}\left(\frac{\partial u}{\partial y}\right)^{2}+\frac{\alpha \sigma B_{0}^{2} u^{2}}{k} \\
u \frac{\partial C}{\partial x}+v \frac{\partial C}{\partial y}=D_{B}\left(\frac{\partial^{2} C}{\partial y^{2}}\right)+\left(\frac{D_{T}}{T_{\infty}}\right)\left(\frac{\partial^{2} T}{\partial y^{2}}\right) \\
u \frac{\partial n}{\partial x}+v \frac{\partial n}{\partial y}+\frac{b W_{c}}{\left(C_{w}-C_{\infty}\right)}\left[\frac{\partial}{\partial y}\left(n \frac{\partial C}{\partial y}\right)\right]=D_{n}\left(\frac{\partial^{2} n}{\partial y^{2}}\right)
\end{gathered}
$$

The corresponding boundary conditions are taken as:

$$
\begin{aligned}
& \left\{\begin{array}{l}
v=0, u=U_{W}(x) \\
-\frac{\partial T}{\partial y}=h_{s}(x) T, C=C_{W}, n=n_{w}
\end{array}\right\}: \text { at } y=0, \\
& \left\{\begin{array}{l}
v \rightarrow 0, u \rightarrow 0, T \rightarrow T_{\infty} \\
C \rightarrow C_{\infty}, n \rightarrow n_{\infty}
\end{array}\right\}: \text { at } y \rightarrow \infty
\end{aligned}
$$

Where $h_{s}$ is the heat transfer parameter. To attain similarity solution of equations (1)-(4), the stream function and dimensionless variables can be posited in the following form:

$$
\begin{array}{r}
\psi=\alpha R a_{x}^{1 / 4} f(\eta), \eta=\frac{y}{x} R a_{x}^{1 / 4}, \theta(\eta)=\frac{T-T_{\infty}}{T_{\infty}} \\
R a_{x}=\frac{\left(1-C_{\infty}\right) \beta g \rho_{f \infty} T_{\infty} x^{3}}{\alpha v}, \beta(\eta)=\frac{C-C_{\infty}}{C_{W}-C_{\infty}}, \phi(\eta)=\frac{n-n_{\infty}}{n_{W}-n_{\infty}}
\end{array}
$$

By applying the similarity transforms on equations (1)(4), the similarity equations are obtained as follows:

$$
\begin{gathered}
f^{\prime \prime \prime}+\frac{3}{4 \operatorname{Pr}} f f^{\prime \prime}-M f^{\prime}+\theta-N r \beta-R b \phi-A f^{\prime}=0 \\
\theta^{\prime \prime}+\frac{3}{4} f \theta^{\prime}+N b \beta^{\prime} \theta^{\prime}+N t \theta^{2}+E c \operatorname{Pr}\left(f^{\prime \prime 2}+M f^{\prime 2}\right)=0 \\
\beta^{\prime \prime}+\frac{N t}{N b} \theta^{\prime \prime}+\frac{3}{4} \operatorname{Le} f \beta^{\prime}=0 \\
\phi^{\prime \prime}+\frac{3}{4} \operatorname{Lbf} \phi^{\prime}-\operatorname{Pe}\left[\beta^{\prime} \phi^{\prime}+\beta^{\prime \prime}(\sigma+\phi)\right]=0
\end{gathered}
$$

Subject to the dimensionless boundary conditions:

$$
\begin{aligned}
& \text { At } \eta=0:\left\{\begin{array}{l}
f=0, f^{\prime}=1, \\
\theta^{\prime}=-\gamma(1+\theta), \beta=1, \phi=1
\end{array}\right\} \\
& \text { At } \eta \rightarrow \infty: f^{\prime}=0, \theta=0, \beta=0, \phi=0
\end{aligned}
$$

Where primes denote differentiation with respect to $\eta$. The parameters of the equation (8)-(11) are defined by:

$$
\begin{aligned}
& \mathrm{P} e=\frac{b W_{c}}{D_{n}}, L e=\frac{\alpha}{D_{B}}, L b=\frac{\alpha}{D_{n}}, A=\frac{x^{2}}{\kappa R a_{x}^{1 / 2}} \\
& N b=\frac{(\rho c)_{P} D_{B}\left(\phi_{W}-\phi_{\infty}\right)}{(\rho c)_{f} \alpha}, N t=\frac{(\rho c)_{P} D_{T}}{(\rho c)_{f} \alpha} \\
& \sigma=\frac{n_{\infty}}{n_{w}-n_{\infty}}, M=\frac{\sigma B_{0}^{2} x^{2}}{v \rho_{f} R a_{x}^{1 / 2}}, E c=\frac{\alpha^{2} R a_{x}}{x^{2} C_{p} T_{\infty}} \\
& R b=\frac{\gamma\left(n_{w}-n_{\infty}\right) \Delta \rho}{\rho_{f} \beta\left(1-C_{\infty}\right) T_{\infty}}, N r=\frac{\left(\rho_{p}-\rho_{f}\right) \Delta C_{w}}{\rho_{f} \beta\left(1-C_{\infty}\right) T_{\infty}}
\end{aligned}
$$

Where $\operatorname{Pr}$ is the Prandtl number, Le is the traditional Lewis number, $\mathrm{Pe}$ is the bioconvection Peclet number, $\mathrm{M}$ is the modified magnetic parameter, Ec is the modified Eckert number, $\mathrm{Nr}$ is the buoyancy ratio parameter, $\mathrm{A}$ is the permeability parameter, $\mathrm{Rb}$ is the bioconvection Rayleigh number, $\mathrm{Lb}$ is the bioconvection Lewis number, $\sigma$ is the bioconvection constant, $\mathrm{Nb}$ is the Brownian motion parameter and $\mathrm{Nt}$ is the thermophoresis parameter.

\section{Results and discussion}

The equations (8)-(11) subject to the boundary conditions (12) have been solved numerically for various range of Newtonian heating boundary condition and for different values of the permeability parameter, Prandtl number, the Lewis number, the buoyancy ratio parameter, the bioconvection constant and bioconvection Rayleigh number. Highly non-linear momentum boundary layer equation and thermal boundary layer equation are converted into similarity equations and then solved numerically by employing fifth order Runge-KuttaFehlberg scheme with shooting method [20].

The most crucial factor of this numerical solution is to choose the appropriate finite value of $\eta_{\infty}$. Thus, the asymptotic boundary conditions given by (15) were replaced by a comparatively large value $\eta_{\max }=15$ for the similarity variable $\left(\eta_{\max }\right)$. The choice of $\eta_{\max }=15$ ensured that all numerical solutions approached to the asymptotic values correctly. It is worth mentioning to consider that the selection of a large value for $\eta_{\max }$ is an important point that is often overlooked in publications on the boundary layer flows. The details of the solution method can be found in [21].

Figure 2 shows the effect of permeability parameter and Newtonian heating parameter on the velocity field in the presence of bioconvection and nanofluid parameters. This figure illustrates that for increased the value of permeability parameter is to decrease the velocity distribution and consequently reduces the thickness of hydrodynamic boundary layer. This happens because of the presence of a porous medium that causes higher 
darcian drag force acting on the nanofluid and induce a deceleration in the flow. Furthermore, it is observed that dimensionless velocity rises in the boundary layer with increasing of the Newtonian heating parameter. This occurs due to the Newtonian heating decreases the density of nanofluid and as a result, the dimensionless velocity increases within the boundary layer.

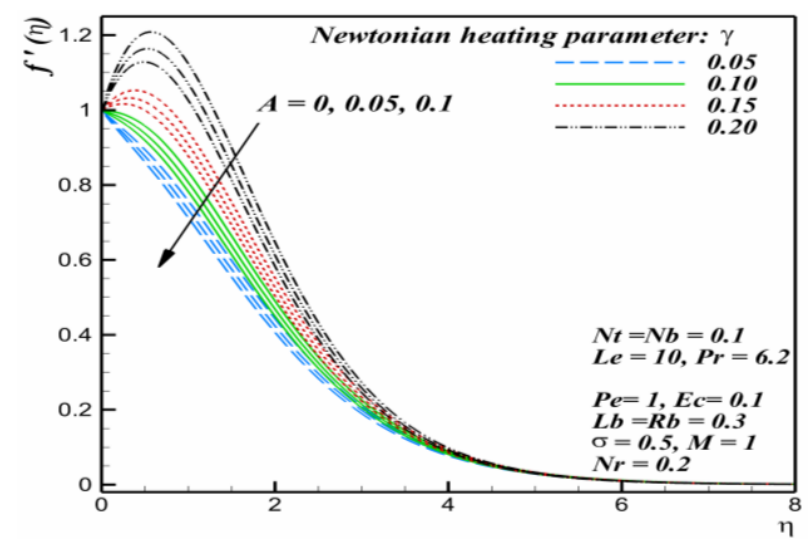

Figure 2. Effects of different parameters on dimensionless velocity

The influence of the buoyancy ratio parameter and Prandtl number on the velocity distribution of bioconvection nanofluid for a stretching sheet is presented in Figure 3. It is found that the dimensionless velocity increases with Prandtl number. The profile of dimensionless temperature for various values of parameter $\mathrm{A}$ and $\mathrm{Y}$ is shown in Figure 4.

Figure 5 depict the influence of the permeability parameter and Lewis number on the dimensionless concentration. Figure 5 shows that the increase in the values of permeability parameter results in the increase in the concentration distribution in the boundary layer region. Also, nanoparticle volume fraction distributions decelerate with the increasing values of the Lewis number in the entire boundary layer region. The Lewis number represents the ratio of the thermal diffusivity to the mass diffusivity. Increasing the Lewis number means a higher thermal diffusivity and a lower mass diffusivity, and this produces thinner concentration boundary layer.

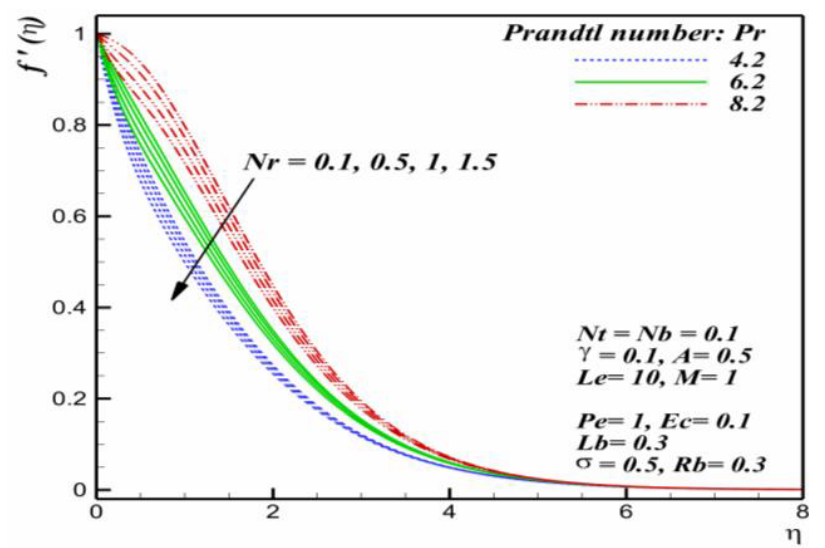

Figure 3. Effects of different parameters on dimensionless velocity.

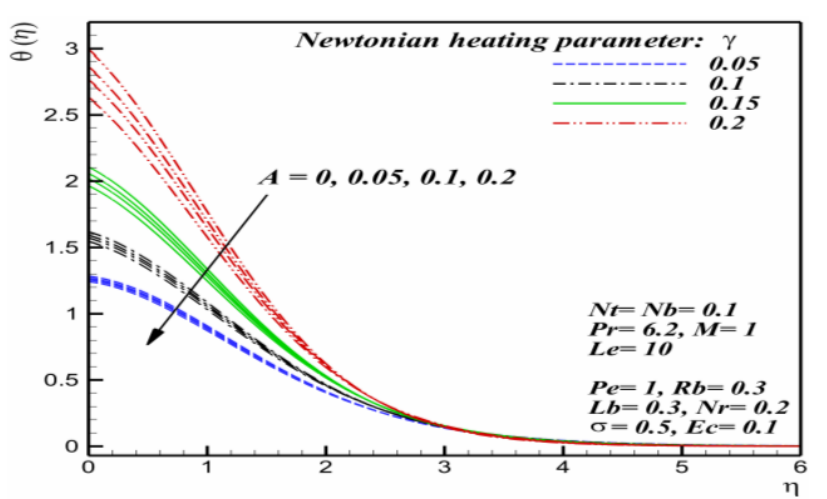

Figure 4. Effects of different parameters on dimensionless temperature

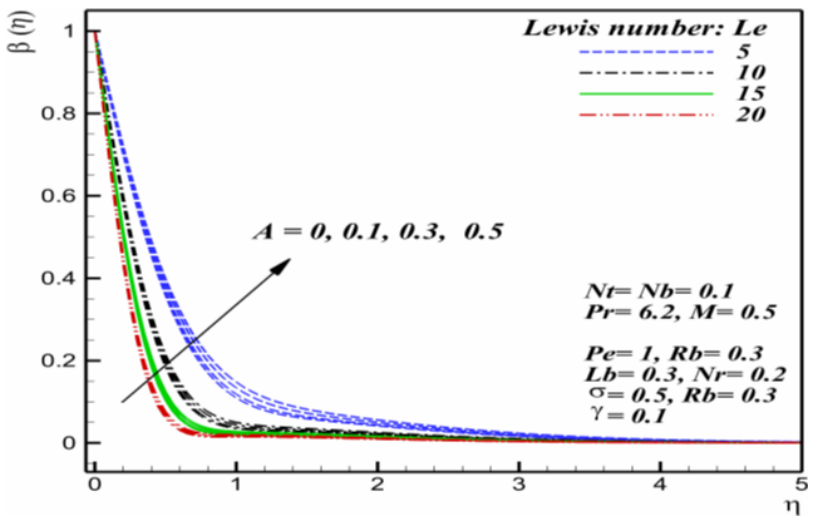

Figure 5. Effects of different parameters on dimensionless concentration.

The effect of bioconvection Rayleigh numbers and Lewis number on the rescaled density of motile microorganisms are shown in Figures 6 and 7. The variation of rescaled density of motile microorganisms respect to bioconvection Raylegh number for different values of bioconvection constant are plotted in Figure 6 . It can be seen that the density of motile microorganisms profile is relatively sensitive and strongly influenced by the changes in $\mathrm{Rb}$ and $\sigma$ parameters. These graphs reveal that an increase in $\sigma$ decreases the concentration thickness for the dimensionless density of motile microorganisms as well as the density of motile microorganisms. On the other hand, the dimensionless density of motile microorganisms increases with increase $\mathrm{Rb}$.

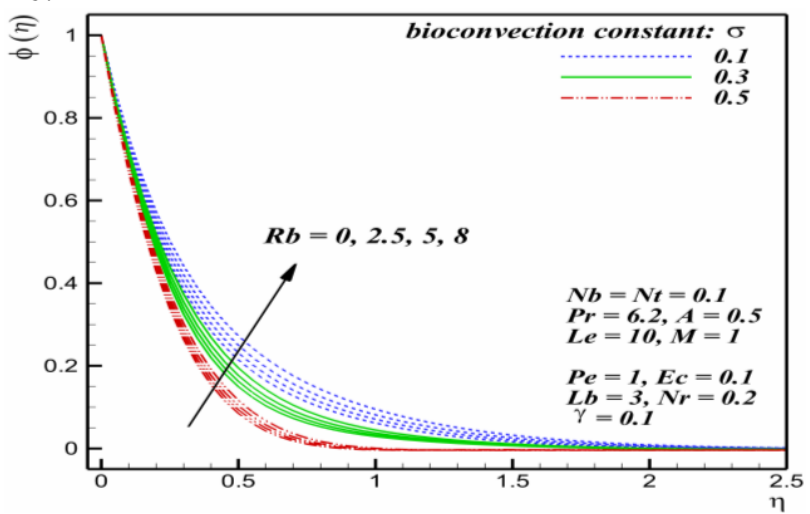

Figure 6. Effects of different parameters on rescaled density of motile microorganisms. 


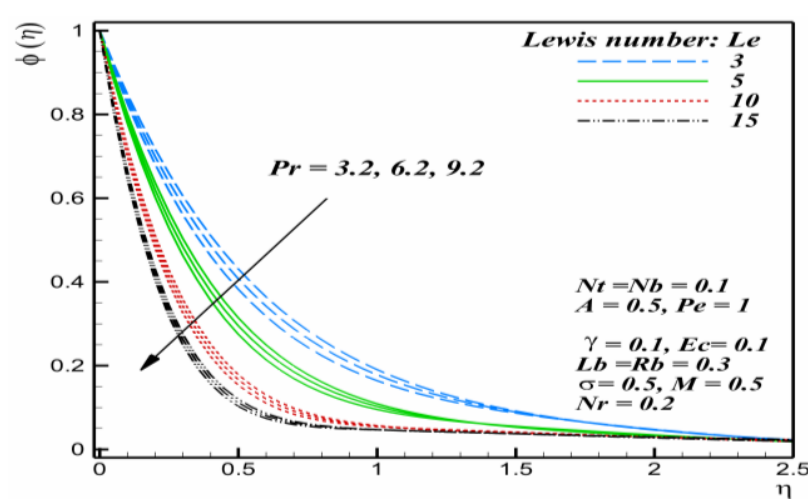

Figure 7. Effects of different parameters on rescaled density of motile microorganisms.

\section{Conclusions}

MHD laminar boundary layer flow with heat and mass transfer on the bioconvection of electrically conducting incompressible nanofluid containing gyrotactic microorganisms past a vertical stretching sheet in a porous medium with Newtonian heating boundary condition are investigated numerically. Based on the results, the significant findings are as follows:

- Dimensionless velocity decreases with Permeability parameter and Buoyancy ratio parameter whereas it increases with Newtonian heating parameter and Prandtl number

- The increase of the Permeability parameter and Lewis number tends to decrease temperature and concentration. But the concentration and temperature increase with increasing Permeability parameter and Newtonian heating parameter.

- The rescaled density of motile microorganisms decreases with Lewis number, Prandtl number and bioconvection constant $(\sigma)$ and increase with increasing bioconvection Rayleigh numbers should be centred and should be numbered with the number on the right-hand side.

\section{References}

1. K. Khanafer, K., Vafai, The Role of Nanoparticle Suspensions in Thermo/Fluid and Biomedical Application. In Nanoparticle Heat Transfer and Fluid Flow, Minkowycz, W. J. Sparrow, E. M. \& Abraham. J. P. (eds.), CRC Press (2012).

2. D. A., Nield, A., Bejan: Convection in Porous Media. Springer, New-York (2013)

3. M. Shafahi, H. Bianco, K. Vafai, O. Manca, An investigation of the thermal performance of cylindrical heat pipes using nanofluids, Int $\mathrm{J}$ of Heat Mass Transf, 55, (2010).

4. K. Vafai, Porous media: applications in biological systems and biotechnology, CRC Press, (2010).

5. D. Huh, B. D. Matthews, A. Mammoto, M. Montoya-Zavala, H. Y. Hsin, D. E. Ingber, Reconstituting organ-level lung functions on a chip, Science, 25, (2010).

6. S. U. S. Choi, Enhancing thermal conductivity of fluids with nanoparticles, ASME-Publications-Fed, 231, (1995).

7. M. Chandrasekar, S. Suresh, T. Senthilkumar, Mechanisms proposed through experimental investigations on thermophysical properties and forced convective heat transfer characteristics of various nanofluids-A review, Renewable and Sustainable Energy Reviews, 16, (2012).

8. A. Noghrehabadi, R. Pourrajab, Experimental investigation of forced convective heat transfer enhancement of $\gamma$-A12O3/water nanofluid in a tube, $\mathrm{J}$ of Mech Sci Tech, 30, (2016).

9. W. Daungthongsuk, S. Wongwises, A critical review of convective heat transfer of nanofluids, Renew Sustainable Energy Review, 11, (2007).

10. A. Noghrehabadi, R. Pourrajab, M. Ghalambaz, Effect of partial slip boundary condition on the flow and heat transfer of nanofluids past stretching sheet prescribed constant wall temperature, Int J of Therm Sci, 54, (2012).

11. A. Noghrehabadi, M. R. Saffarian, R. Pourrajab, M. Ghalambaz, Entropy analysis for nanofluid flow over a stretching sheet in the presence of heat generation/absorption and partial slip, J of Mech Sci Tech, 27, (2013).

12. A. Noghrehabadi, R. Pourrajab, M. Ghalambaz, Flow and heat transfer of nanofluids over stretching sheet taking into account partial slip and thermal convective boundary conditions, Heat and Mass Transfer, 49, (2013).

13. A. Noghrehabadi, M. Ghalambaz, E. Izadpanahi, R. Pourrajab, Effect of magnetic field on the boundary layer flow, heat, and mass transfer of nanofluids over a stretching cylinder, $\mathrm{J}$ of Heat and Mass Transf Research, 1, (2014).

14. A. Noghrehabadi, R. Pourrajab, E. Izadpanahi, Similarity solution of boundary layer flow over a stretching cylinder saturated with a nanofluid, Int J of Energy Clean Environment, 16, (2016).

15. J. Buongiorno, Convective transport in nanofluids, $\mathrm{J}$ of Heat Transfer, 128, (2006).

16. A. V Kuznetsov, The onset of nanofluid bioconvection in a suspension containing both nanoparticles and gyrotactic microorganisms, Int Comm in Heat and Mass Transfer, 37, (2010).

17. A. V. Kuznetsov, D. A. Nield, The ChengMinkowycz problem for natural convective boundary layer flow in a porous medium saturated by a nanofluid: a revised model, Int $\mathrm{J}$ of Heat and Mass Transfer, 65, (2013).

18. D. A. Nield, A. V. Kuznetsov, The ChengMinkowycz problem for the double-diffusive natural convective boundary layer flow in a porous medium saturated by a nanofluid, Int $\mathrm{J}$ of Heat and Mass Transfer, 54, (2011).

19. W. A. Khan, O. D. Makinde, MHD nanofluid bioconvection due to gyrotactic microorganisms over a convectively heat stretching sheet, Int $\mathrm{J}$ of Therm Sci, 15, (2014).

20. T.Y. Na, Computational Method in Engineering Boundary Value Problems, Academic Press, New York, (1979).

21. M. L. F. Shampine, J. Kierzenka, M. W. Reichelt, Solving boundary value problems for ordinary differential equations in MATLAB with bvp4c, Tutorial notes, (2000). 